\title{
A multi-center randomized controlled trial of the LenoMed ATA-I-1-0 insulin pump for the intensive treatment of diabetic patients
}

\author{
Jin Du ${ }^{1 \#}$, Li Zang ${ }^{1 \#}$, Yufeng $\mathrm{Li}^{2 \#}$, Jia Liu ${ }^{3}$, Lijuan Wang ${ }^{2}$, Yan Duan ${ }^{3}$, Guangxia Guo ${ }^{2}$, Yijun $\mathrm{Li}^{1}$, \\ Yajing Zhang', Guang Wang ${ }^{3}$, Yiming $\mathrm{Mu}^{1}$
}

${ }^{1}$ Department of Endocrinology, The First Medical Center of PLA General Hospital, Beijing, China; ${ }^{2}$ Department of Endocrinology, Beijing Pinggu Hospital, Beijing, China; ${ }^{3}$ Department of Endocrinology, Beijing Chaoyang Hospital, Capital Medical University, Beijing, China

Contributions: (I) Conception and design: J Du, L Zang, Y Li, G Wang, Y Mu ; (II) Administration support: G Wang, Y Mu; (III) Provision of study materials or patients: L Wang, Y Duan, G Guo; (IV) Collection and assembly of data: Y Li, Y Zhang; (V) Data analysis and interpretation: J Du, L Zang, Y Li; (VI) Manuscript writing: All authors; (VII) Final approval of manuscript: All authors.

\#These authors contributed equally to this work.

Correspondence to: Guang Wang. Department of Endocrinology, Beijing Chaoyang Hospital, Capital Medical University, 8 Gongtinan Road, Chaoyang District, Beijing 100020, China. Email: drwg6688@aliyun.com; Yiming Mu. Department of Endocrinology, The First Medical Center of PLA General Hospital, 28 Fuxing Road, Haidian District, Beijing 100853, China. Email: muyiming@301hospital.com.cn.

Background: Continuous subcutaneous insulin infusion (CSII) is an effective method for managing diabetes. The aim of this study was to evaluate the efficacy and safety of the LenoMed ATA-I-1-0 insulin pump for the treatment of patients with type 1 and type 2 diabetes, compared to the Medtronic MMT-712 insulin pump.

Methods: A total of 120 subjects with diabetes needing insulin treatment were recruited, and 104 completed the trial. The intervention and control group used the LenoMed and Medtronic insulin pump, respectively. The primary outcome was a reduction in fasting plasma glucose. Secondary outcomes included blood glucose control at 7 time points (before and after each meal, and before sleep), hemoglobin A1c (HbA1c) levels at 1 time point, and accuracy and safety of the pumps.

Results: In the per protocol set of 104 subjects, a significant drop in fasting plasma glucose levels was observed for $98.0 \%$ subjects in the intervention group and $92.7 \%$ in the control group. There was no statistically significant difference between groups [5.23\%; 95\% confidence interval (CI): $-2.69 \%-13.2 \%$ ]. Compared to baseline, levels of blood glucose and HbA1c dropped $(\mathrm{P}<0.001)$ at a similar magnitude in both groups. The accuracy and alarm effectiveness was 100\%. No adverse event or severe adverse event related to the medication occurred in the study.

Conclusions: The novel injection equipment, the LenoMed ATA-I-1-0 insulin pump, demonstrated efficacy and a good safety profile, and was not inferior to the widely used Medtronic MMT-712 insulin pump. Thus, our study suggests that the LenoMed ATA-I-1-0 insulin pump can be widely used in clinical practice.

Keywords: Randomized controlled trial; LenoMed ATA-I-1-0; insulin pump; type 1 diabetes; type 2 diabetes

Submitted Jul 24, 2020. Accepted for publication Oct 26, 2020.

doi: 10.21037/atm-20-6024

View this article at: http://dx.doi.org/10.21037/atm-20-6024 


\section{Introduction}

According to the International Diabetes Federation, 463 million people worldwide were living with diabetes in 2019, with the highest proportion in China (116 million), representing approximately a quarter of the global population with diabetes (1). In addition, the prevalence of diabetes in China has drastically increased over the past 30 years (2). According to results from epidemiological studies, the prevalence of diabetes increased from $9.7 \%$ in 2008 to $10.9 \%$ in 2013 , with $60 \%$ of individuals unaware of their diagnosis (3). Moreover, another $35.7 \%$ already had abnormal glucose levels, and were at higher risk of developing diabetes (3). Therefore, the intervention and treatment of diabetes are of the utmost importance in China.

The treatment goal of diabetes is to help patients maintain glycemic level within a near-normal target range, and the treatment process is case-dependent. Some patients with diabetes can receive oral medication or premixed insulin treatment. However, others require multiple daily injection (MDI) of short-acting/fast-acting insulin combined with long-acting insulin to maintain good control of plasma glucose levels, which also helps to avoid the occurrence and development of complications, particularly for patients with type 1 diabetes, a long duration of type 2 diabetes coupled with insulin failure and severe hyperglycemia, or gestational diabetes (4). These patients must receive an average of 1,440 injections every year with MDI, which severely affects their quality of life.

Continuous subcutaneous insulin infusion (CSII) is an alternative method to MDI. CSII pumps can more effectively stimulate physiological insulin secretion, continuously providing insulin to the body at the right time (5). In 2004, the American Diabetes Association suggested that CSII is an effective means of managing diabetes with the goal of achieving near-normal levels of blood glucose and improving lifestyle flexibility (6). Evidence from large trials has also shown that compared to MDI, CSII achieves lower hemoglobin A1c (HbA1c) levels with a reduced rate of hypoglycemia (7), indicating that CSII is a more effective method for managing diabetes. Additionally, CSII is more convenient to use than MDI, meaning patients treated with CSII have higher satisfaction and a better quality of life than those treated with MDI (8). In general, patients who need intensive insulin therapy may consider using insulin pumps.
CSII pumps were first invented in the United Kingdom (9). It was estimated that in 2002, more than 200,000 patients worldwide with diabetes used CSII pumps (10). CSII insulin pumps entered the Chinese market nearly 20 years ago. At present, approximately 40,000 individuals are using pumps for long-term treatment. Approximately 3,000 hospitals now have insulin pump treatment, and it is estimated that more than 1 million patients have received short-term insulin pump treatment (11). Currently, the insulin pumps used in clinical practice in China are mainly overseas brands. Restricted by factors such as price, purchase channels, and after-sales service, many diabetic patients who require insulin therapy fail to adopt the insulin pump as a convenient and reliable method of administration. An insulin pump, the LenoMed ATA-I-1-0 model, was produced domestically by LenoMed Corporationg in strict accordance with relevant industry standards and national standards, and quality was approved by the inspection of regulatory departments (Beijing Medical Device Quality Supervision and Inspection Center of the State Food and Drug Administration). Compared to existing domestic insulin pumps, the LenoMed ATA-I-1-0 model is more user-friendly, environmentally friendly, and cost-effective. However, its efficacy and safety have not been assessed in a clinical trial.

Therefore, we conducted a randomized controlled trial to compare the efficacy and safety of the LenoMed ATA-I-1-0 insulin pump with the Medtronic MMT-712 insulin pump, which has been shown to be effective (12) and is widely used in clinical practice in China by patients with type 1 and type 2 diabetes. The duration of the trial was 7 days. The primary outcome was an overall drop in fasting plasma glucose levels. Secondary outcomes included the control of blood glucose levels at 7 time points (before and after each meal, and before sleep), HbA1c levels at 1 time point at the end of the trial (day 7), the accuracy of the pump amount, and safety [effectiveness of the alarm and adverse events (AEs)]. The novel goal of this work was to evaluate the efficacy and safety of the LenoMed ATA-I-1-0 insulin pump in clinical settings. We hypothesized that the LenoMed ATA-I-1-0 insulin pump is non-inferior to the Medtronic MMT-712 insulin pump and can be widely used in clinical practice. We present the following article in accordance with the CONSORT reporting checklist (available at http://dx.doi. org/10.21037/atm-20-6024). 


\section{Methods}

\section{Trial design and participants}

The trial was a multi-center randomized controlled trial across 3 hospitals in Beijing, China (Chinese People's Liberation Army General Hospital, Beijing Chaoyang Hospital, and Beijing Pinggu District Hospital). We recruited patients with diabetes (both type 1 and type 2) who needed intensive insulin therapy. Eligible patients met the following criteria: (I) aged between 18 and 75 years old; (II) patients with type 1 or type 2 diabetes who needed insulin therapy; (III) HbA1c levels $\geq 9.0 \%$; (IV) no change in treatment plan 30 days before hospital admission; and (V) provided voluntary informed consent. Patients were excluded according to the following criteria: (I) patients were not eligible to receive insulin treatment; (II) had hyperglycemia with severe circulation disorders and appeared to be in shock; (III) had severe impairment of heart, liver, and kidney function and other systemic diseases; (IV) had mental illness, no self-control, or could not express themselves clearly; (V) had acute complications of diabetes such as diabetic ketoacidosis and diabetic hypertonic nonketogenic coma; (VI) were breastfeeding, had a positive pregnancy test, or were preparing for pregnancy; (VII) had allergies or were allergic to tape; (VIII) participated in other clinical trials in the last 3 months; or (IX) showed poor compliance. The current study complied with the requirements of the State Food and Drug Administration and the Declaration of Helsinki and its amendments, and respected the rights of participants according to the requirements stipulated in the Good Medical Device Clinical Trial Quality Management Practices. The trial was registered (ChiCTR2000029725), and the protocol was published (13). The institutional ethics committees from Chinese People's Liberation Army General Hospital (Approval No. 2017-023-1), Beijing Chaoyang Hospital (Approval No. 2018-4-17-3), and Beijing Pinggu District Hospital (Approval No. 2017-002-04) all approved the current study. Written informed consent was obtained from all participants prior to enrollment.

\section{Trial groups}

The trial group used the LenoMed ATA-I-1-0 insulin pump (Figure S1), which was produced by LenoMed Corporationg. The basal insulin dose and total daily dose in the trial group were $17.61 \pm 7.71$ units (range, 9.843.2 units) and $43.05 \pm 15.31$ units (range, 24-81.6 units), respectively. The control group used the Medtronic MMT712 insulin pump (Registration No. National Machinery injection 20173541951, Figure S2), which was produced by Medtronic (Shanghai) Management Co., Ltd. (Shanghai, China). The basal insulin dose and total daily dose in the control group were 18.64 \pm 7.52 units (range, 6.9-33.4 units) and $44.89 \pm 16.81$ units (range, 20-82.4 units), respectively. The duration of the intervention was 7 days.

\section{Trial assessment and outcomes}

We assessed the outcomes of 3 population sets. The first set was the full analysis set (FAS), which referred to the dataset consisting of all subjects who participated in treatment at baseline according to the "Intention to Treat" principle. The second set was the per protocol set (PPS), which referred to a subset of the FAS comprising patients who completed the treatment without protocol violation. The third set was the safety analysis set (SAS), composed of recruited patients who completed at least 1 treatment and were used for the safety evaluation.

\section{Efficacy evaluation}

The primary outcome of the current trial was overall reduction in fasting plasma glucose levels after the trial by comparing fasting plasma glucose levels between prepump and day 7 of pump therapy. Secondary outcomes included overall reduction in blood glucose levels at 7 time points (before and after each meal, and before sleep) and HbA1c levels at 1 time point at the end of the trial (day 7). Blood samples $(3 \mathrm{~mL})$ were used for the measurement of blood glucose levels using the hexokinase method and a range between 3.9 and $6.1 \mathrm{mmol} / \mathrm{L}$ was considered normal. Blood samples $(2 \mathrm{~mL})$ were collected for the assessment of HbA1c levels using the high-performance liquid chromatographic method and a range between $4.0 \%$ and $6.0 \%$ was considered normal. The accuracy of the pump amount was evaluated by comparing the actual pump amount with the doctor's prescription.

\section{Safety evaluation}

We evaluated the effectiveness of the alarm by recording the alarm status daily during use, observing whether the alarm was triggered when the alarm condition was met, and whether the condition that triggered the alarm met the criteria. Alarm validity $\geq 80 \%$ was regarded as effective. We also recorded the number and occurrence of AEs and serious AEs. 


\section{Applicability evaluation}

The subjective evaluation of clinical applicability of the product included "marks", "operation", "product stability", "overall design (including appearance, shape)", and "comfort." The clinical applicability evaluation was a secondary evaluation and did not affect the safety and efficacy analysis of the product. Evaluation levels were scored according to different levels of satisfaction, ranging from very satisfied ( 9 points) to very dissatisfied ( 0 points). Three or more evaluations $<6$ points indicated poor clinical applicability, otherwise the clinical applicability was considered good.

\section{Statistical analysis}

The estimated total sample size was 120 , under the assumption that the efficacy of insulin pumps in both groups was $97 \%$ and the non-inferiority threshold $\delta$ was $10 \%$, with an $80 \%$ retention rate and a 2 -sided type I error rate of $5 \%$. Therefore, the respective sample size was 60 for the control and intervention groups. All statistical tests were 2 -sided. $\mathrm{P}$ values less than 0.05 were considered statistically significant. Continuous variables are presented as the mean and standard deviation or median and interquartile range, and categorical variables are presented as the number of cases and percentages.

Baseline characteristics (e.g., age, height, body weight) between the control and intervention groups were compared to ensure comparability between groups. The comparison of continuous variables between the control and intervention groups was conducted using a Student's $t$-test or Wilcoxon rank-sum test. Categorical variables were compared using the chi-square test or Fisher's exact probability test. Ordinal variables were compared using the Wilcoxon rank-sum test or Cochran-Mantel-Haenszel test. Statistical analyses were performed in the FAS, PPS, and SAS datasets. If the lower $95 \%$ confidence interval (CI) of the difference of blood glucose levels between the intervention and control groups was $\geq-10 \%$, the insulin pump used in the intervention group was regarded as being non-inferior to that of the control group.

\section{Results}

\section{Participants}

A total of 120 patients were screened, recruited, and randomized for the current trial, and 104 completed the trial. Among the 120 patients, 3 retrieved the consent form before the treatment, and the other 3 were excluded due to: presenting with ketosis and being intravenously injected with insulin; not meeting the requirement for intensive insulin therapy; or presenting with anxiety before the study. Therefore, 114 patients received treatment (54 in the intervention group and 60 in the control group), and 112 patients completed the treatment (53 in the intervention group and 59 in the control group). Among the 112 patients, 8 patients had protocol deviations and were further excluded. A detailed description of the patients stratified by the 3 hospitals is shown in Table S1. In addition, the FAS and SAS sets had 112 and 114 patients, respectively, and the PPS set had 104 patients (Tables S2,S3). The main patient characteristics between the control and intervention groups are shown in Table 1 . The mean age in this study was 49.25 (14.60) years; $65.79 \%$ were men and $94.74 \%$ were of Han ethnicity. The control and intervention groups had similar demographic factors (age, gender, ethnicity), lifestyle (smoking, alcohol consumption, and physical activity levels), medical history (type of diabetes, and complications with diabetes), and clinical data (weight, pulse, blood pressure) (all $\mathrm{P}>0.05$ ) except for the control group, which had a higher rate of co-morbidities $(\mathrm{P}=0.045)$. The basal insulin dose and total daily dose between the two groups were not statistically significant $(\mathrm{P}=0.477$ and $\mathrm{P}=0.457$, respectively).

\section{Trial outcomes}

In the PPS, the baseline mean fasting plasma glucose levels were 11.9 (2.81) $\mathrm{mmol} / \mathrm{L}$ and 11.9 (3.59) $\mathrm{mmol} / \mathrm{L}$ for the intervention and control group, respectively. After using the insulin pumps, the mean fasting plasma glucose levels significantly dropped in both the intervention [6.31 (1.72) $\mathrm{mmol} / \mathrm{L}]$ and control group [5.94 (1.81) $\mathrm{mmol} / \mathrm{L}]$ (Table 2). The mean levels of fasting plasma glucose were similar before ( $\mathrm{P}=0.860$ in FAS, $\mathrm{P}=0.915$ in PPS) and after the intervention $(\mathrm{P}=0.418$ in FAS, $\mathrm{P}=0.193$ in PPS) between the 2 groups. In the PPS dataset, the efficacy of the insulin pump treatment was $98.0 \%$ and $92.7 \%$ in the intervention and control group, respectively, and the difference in efficacy between both groups was 5.23\% (95\% CI: $-2.69 \%$ to $13.15 \%)$. Because the lower $95 \%$ CI was higher than $-10 \%$, the LenoMed ATA-I-1-0 insulin pump used in the intervention group was non-inferior to the Medtronic MMT-712 insulin pump used in the control group. The non-inferior result was similar when using the FAS dataset [6.30\% (95\% CI: $-2.81 \%$ to $15.41 \%)$ ], suggesting that 
Table 1 Baseline characteristics of the study population

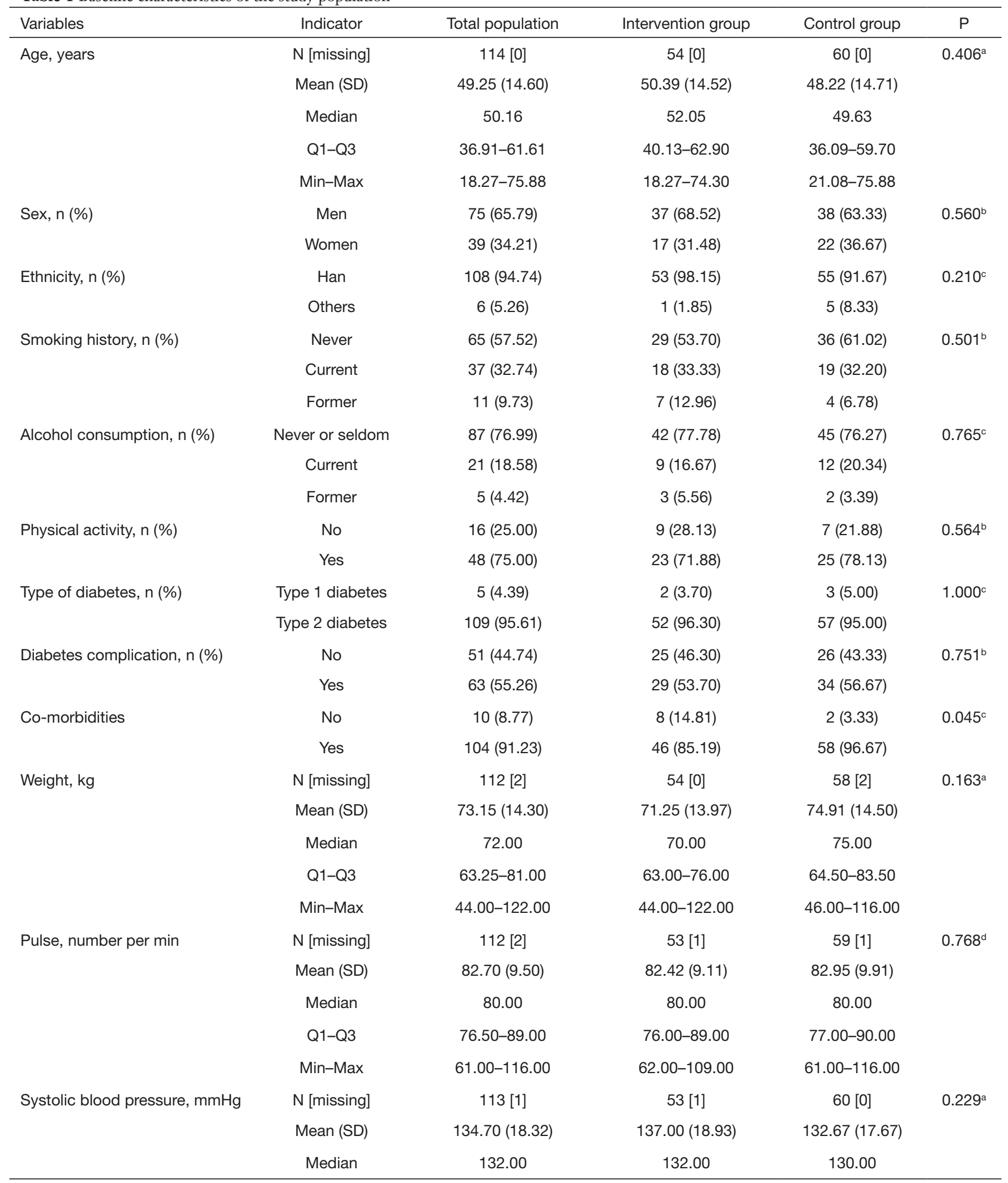

Table 1 (continued) 
Table 1 (continued)

\begin{tabular}{|c|c|c|c|c|c|}
\hline Variables & Indicator & Total population & Intervention group & Control group & $\mathrm{P}$ \\
\hline & Min-Max & $99.00-200.00$ & 104.00-200.00 & $99.00-185.00$ & \\
\hline \multirow[t]{4}{*}{ Diastolic blood pressure, $\mathrm{mmHg}$} & $\mathrm{N}$ [missing] & $113[1]$ & $53[1]$ & $60[0]$ & $0.993^{\mathrm{a}}$ \\
\hline & Mean (SD) & $82.01(11.66)$ & $83.34(12.20)$ & $80.83(11.12)$ & \\
\hline & Q1-Q3 & $76.00-89.00$ & $76.00-89.00$ & $78.00-88.50$ & \\
\hline & Min-Max & $41.00-126.00$ & $60.00-126.00$ & $41.00-100.00$ & \\
\hline
\end{tabular}

${ }^{a}$, the comparison was conducted using the Wilcoxon rank-sum test; ${ }^{b}$, the comparison was conducted using the chi-square test; ${ }^{c}$, the comparison was conducted using Fisher's exact probability test; ${ }^{d}$, the comparison was conducted using Student's $t$-test. SD, standard deviation; $\mathrm{Q}$, quartile.

Table 2 Fasting plasma glucose levels before treatment in the control and intervention groups

\begin{tabular}{|c|c|c|c|c|c|}
\hline Variable & Indicator & \multicolumn{2}{|c|}{ Full analysis set } & \multicolumn{2}{|c|}{ Per protocol set } \\
\hline \multirow[t]{5}{*}{ Baseline } & $\mathrm{N}$ [missing] & $54[0]$ & $59[1]$ & $49[0]$ & $55[0]$ \\
\hline & Mean (SD) & $12.08(2.80)$ & 11.97 (3.53) & $11.88(2.81)$ & $11.94(3.59)$ \\
\hline & Median & 11.99 & 12.24 & 11.27 & 12.24 \\
\hline & Min-Max & $7.36-21.17$ & $4.96-19.77$ & $7.36-21.17$ & $4.96-19.77$ \\
\hline & $P$ value ${ }^{a}$ & 0.860 & & 0.915 & \\
\hline \multirow[t]{4}{*}{ Day 7} & $\mathrm{~N}$ [missing] & $53[1]$ & $59[1]$ & $49[0]$ & $55[0]$ \\
\hline & Mean (SD) & $6.26(1.67)$ & $6.06(1.83)$ & $6.31(1.72)$ & $5.94(1.81)$ \\
\hline & Min-Max & $3.88-12.44$ & $3.00-14.01$ & $3.88-12.44$ & $3.00-14.01$ \\
\hline & $P$ value ${ }^{b}$ & 0.418 & & 0.193 & \\
\hline
\end{tabular}

${ }^{a}$, the comparison was conducted using Student's $t$-test; ${ }^{b}$, the comparison was conducted using the Wilcoxon rank-sum test.

the LenoMed ATA-I-1-0 insulin pump can be effectively used in clinical practice (Table 3). The baseline glucose levels at 7 time points during the day (before and after each meal, and before sleep) significantly decreased at the corresponding time points on day 7 in both the intervention and control group (Figure 1 and Tables S4-S10). The glucose levels were comparable between the intervention and control group before and after treatment, except for 1 time point after the treatment for which the intervention group had lower glucose levels (before sleep in
FAS; $\mathrm{P}=0.024)$. Similarly, the HbA1c levels also dropped at the end of the trial, and there was no statistical difference in HbA1c levels before or after treatment between the control and intervention group (Table S11). The accuracy of the LenoMed ATA-I-1-0 insulin pump was 100\% (Table S12). No AEs (e.g., skin infections) or serious AEs occurred during this clinical trial. The AEs that occurred were not related to the insulin pumps, and there was no statistical difference between the control and intervention groups. The practicability of insulin pumps used in the intervention 
Table 3 Differences between fasting plasma glucose levels before and after treatment in the intervention and control groups

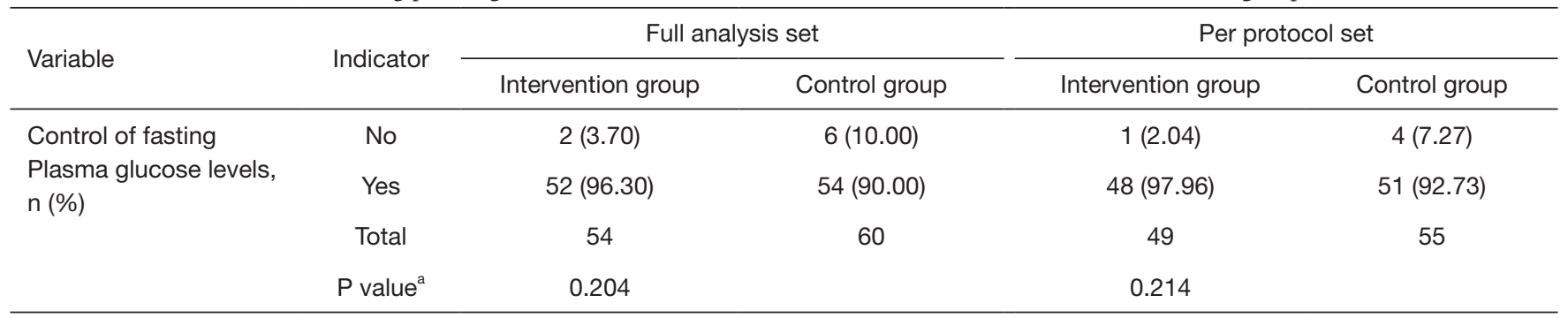

${ }^{\mathrm{a}}$, the comparison was conducted using the Cochran-Mantel-Haenszel test.
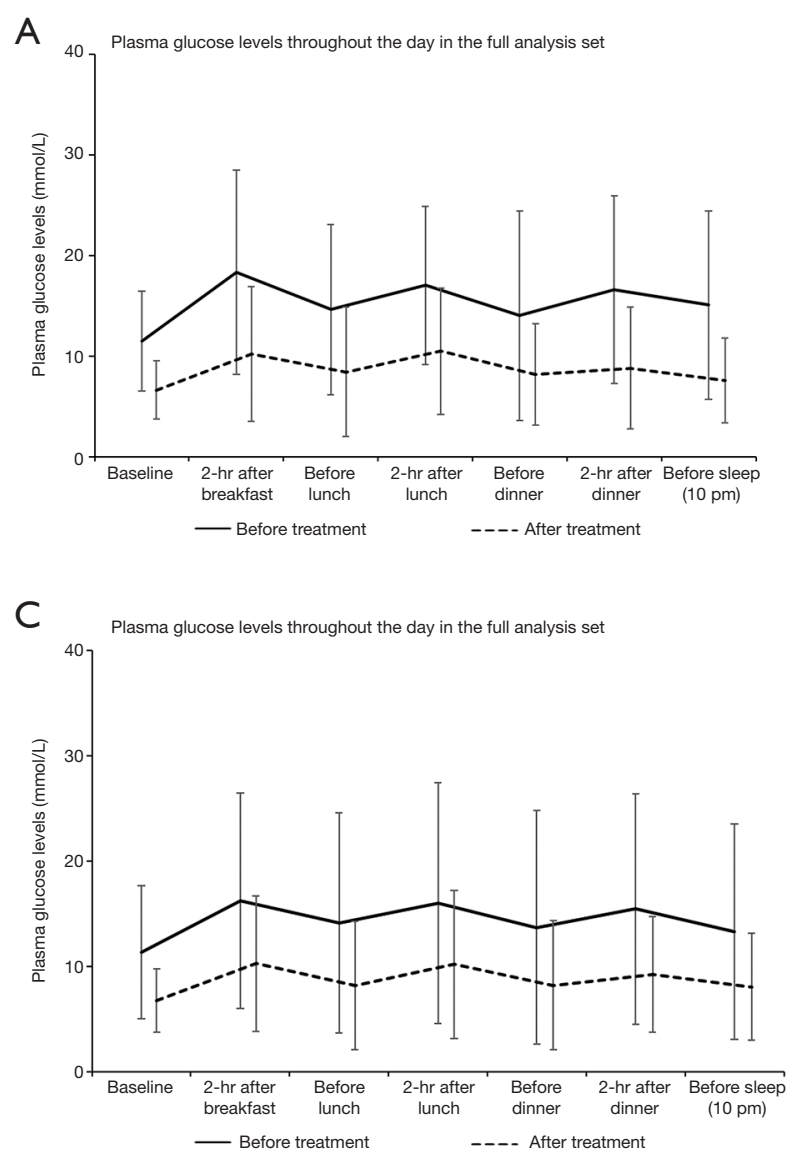

Figure 1 Plasma glucose levels at 7 time points (mean $\pm 1.96 \times$ standard deviation) during the day prior to and after treatment in the intervention group $(A, B)$ and the control group $(C, D)$.

and control group were both $100.00 \%$ and did not differ between groups.

\section{Discussion}

In this multi-center randomized controlled trial among patients with diabetes, the scientific problem of whether
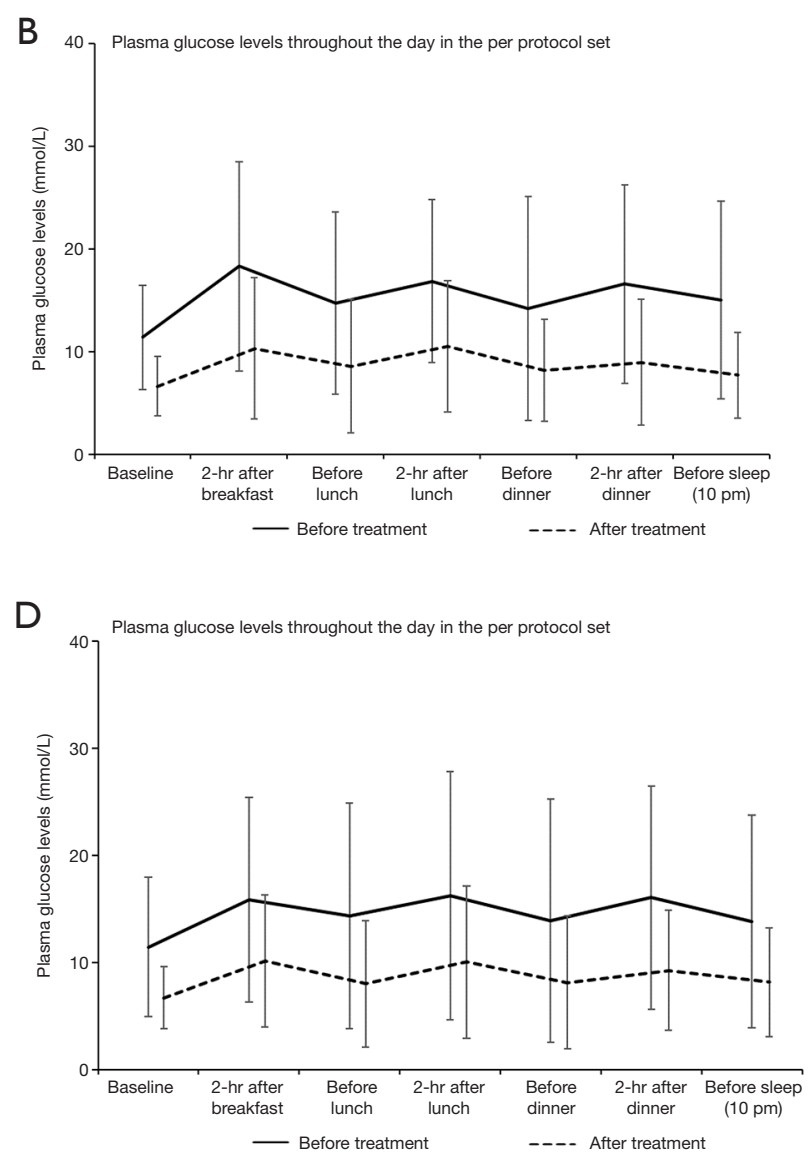
levels at various time points during the day, and a reduction of HbA1c levels. The efficacy of the LenoMed ATA-I-1-0 insulin pump was non-inferior to the insulin pump used in the control group. In addition, the LenoMed ATA-I-1-0 model was demonstrated to be accurate and safe, with clinical applicability. Our results suggest that the LenoMed ATA-I-1-0 insulin pump can be widely applied in clinical practice.

Our results were consistent with evidence from previous clinical trials showing that CSII pumps are effective in decreasing blood glucose levels $(6,7)$. In 2014, the American Diabetes Association confirmed that CSII is effective in diabetes management (6), and large-scale trials demonstrated that CSII is not only effective in reducing blood glucose levels, but also reduces the rate of hypoglycemia, demonstrating that CSII pumps are an effective method for quality diabetes care (7). In the current study, the main indicator for the efficacy of the LenoMed ATA-I-1-0 insulin pump was a difference in fasting plasma glucose levels before and after treatment. The efficacy of the LenoMed ATA-I-1-0 insulin pump was slightly higher than that of the Medtronic MMT-712 insulin pump in the FAS (96.3\% vs. $90.0 \%)$ and PPS (98.0\% vs. $92.7 \%)$ with slightly overlapping $95 \% \mathrm{CI}$, indicating that the LenoMed ATA-I-1-0 model was non-inferior to the Medtronic MMT712 model and meets the requirements for clinical use. The secondary indicators of efficacy were the comparison of plasma glucose levels at 7 time points throughout the day and $\mathrm{HbA} 1 \mathrm{c}$ levels before and after treatment. As a result, all glucose levels and HbA1c levels significantly dropped, and the LenoMed ATA-I-1-0 insulin pump showed a better performance compared to the Medtronic MMT-712 insulin pump for bedtime glucose levels. Similarly, the efficacy of the LenoMed ATA-I-1-0 insulin pump reported in this study was also higher than that of other insulin pumps from China and overseas, such as the Intelligent Insulin Pump by the WEGO group (14), Sheng Tang Insulin Pump (15), Medtronic Paradigm 712 (14), and Medtronic MiniMed 507C (15), which further supports the efficacy of the LenoMed ATA-I-1-0 insulin pump and its use in clinical practice.

In addition, the pump dose of the LenoMed ATA-I-1-0 insulin pump was shown to be stable throughout treatment, and the accuracy was $100 \%$ in accordance with the physician's prescription. Moreover, the LenoMed ATA-I-1-0 insulin pump was safe to use, with an alarm effective rate of $100 \%$ and no associated AEs. In addition, no skin infection was reported at the puncture site, further highlighting its safety profile.

A systematic review and meta-analysis comprising 74 studies showed that the use of CSII was cost-effective (16). Among the existing insulin pumps, the cost of the LenoMed ATA-I-1-0 insulin pump was among the lowest. Compared to the Medtronic MMT-712 insulin pump that was used in the control group (59,8000 RMB), the cost of the LenoMed ATA-I-1-0 insulin pump (19,999 RMB) was approximately a third of the Medtronic MMT-712 insulin pump. In addition, it was also cheaper than other insulin pumps from China and overseas (price ranged between 23,500 RMB and 32,800 RMB). Therefore, the LenoMed ATA-I-1-0 insulin pump was more cost saving to patients. In addition to the efficacy, safety, and cost-effectiveness, the LenoMed ATA-I-1-0 insulin pump also innovatively adopted advanced technology to enhance convenience for users. For the first time, the Organic Light Emitting Diode color screen with a large display and touch function was adopted, which made the visualization clearer and the pump easier to use. In the dose setting, the automatic allocation of basal rate helps to improve the work efficiency of frontline medical staff. In addition, the LenoMed ATA-I-1-0 insulin pump is the only pump equipped with Bluetooth, which helps transfer treatment information to the mobile phone and to the "cloud", and provides an important basis for the network of chronic disease management in various hospitals. Therefore, the advantages of the LenoMed ATA-I-1-0 insulin pump include high efficacy and safety, cost-effectiveness, and userfriendly interface. We did not observe any disadvantages of the LenoMed ATA-I-1-0 insulin pump compared to the Medtronic MMT-712 model. Future studies are needed to validate the efficacy of the LenoMed ATA-I-1-0 insulin pump for long-term diabetes management.

The current study had several strengths. First, we conducted a multi-center randomized controlled trial, and conducted comprehensive statistical analyses. Second, we also used a wide range of indicators to evaluate the efficacy and safety of the intervention. In addition, we utilized a widely used insulin pump as a control group, thereby increasing the clinical implication of the intervention. Moreover, we included patients with both type 1 and type 2 diabetes, which greatly enhanced the generalizability of our intervention. However, this study had some limitations. First, the current study evaluated the shortterm effectiveness of the intervention. Further studies are warranted to evaluate the efficacy of the intervention during a longer period. In addition, the majority of recruited patients were of Han ethnicity; therefore, further studies 
are needed to determine if the results of this study are generalizable to other ethnic groups. In addition, additional studies with a longer study duration, larger sample size, and more ethnic groups are warranted to validate our findings.

\section{Conclusions}

In conclusion, in this multi-center randomized controlled trial of patients with diabetes, we found that the newly developed LenoMed ATA-I-1-0 insulin pump demonstrates efficacy and safety for use in clinical practice, and was noninferior to the insulin pump used in the control group. Our results suggest that the LenoMed ATA-I-1-0 insulin pump can be widely applied in clinical practice. Future studies should be conducted to validate the efficacy of the LenoMed ATA-I-1-0 insulin pump for short- and longterm diabetes management as well as its cost-effectiveness.

\section{Acknowledgments}

Funding: None.

\section{Footnote}

Reporting Checklist: The authors have completed the CONSORT reporting checklist. Available at http://dx.doi. org/10.21037/atm-20-6024

Data Sharing Statement: Available at http://dx.doi. org/10.21037/atm-20-6024

Conflicts of Interest: All authors have completed the ICMJE uniform disclosure form (available at http://dx.doi. org/10.21037/atm-20-6024). The authors have no conflicts of interest to declare.

Ethical Statement: The authors are accountable for all aspects of the work in ensuring that questions related to the accuracy or integrity of any part of the work are appropriately investigated and resolved. The current study complied with the requirements of the State Food and Drug Administration and the Declaration of Helsinki and its amendments, and respected the rights of participants according to the requirements of the Good Medical Device Clinical Trial Quality Management Practices. The trial was registered (ChiCTR2000029725), and the protocol was published 13. The institutional ethics committees from Chinese People's Liberation Army General Hospital
(Approval No. 2017-023-1), Beijing Chaoyang Hospital (Approval No. 2018-4-17-3), and Beijing Pinggu District Hospital (Approval No. 2017-002-04) all approved the current study. Written informed consent was obtained from all participants prior to enrollment.

Open Access Statement: This is an Open Access article distributed in accordance with the Creative Commons Attribution-NonCommercial-NoDerivs 4.0 International License (CC BY-NC-ND 4.0), which permits the noncommercial replication and distribution of the article with the strict proviso that no changes or edits are made and the original work is properly cited (including links to both the formal publication through the relevant DOI and the license). See: https://creativecommons.org/licenses/by-nc-nd/4.0/.

\section{References}

1. International Diabetes Federation. IDF Western Pacific members 2019. Available online: https://idf.org/ournetwork/regions-members/western-pacific/members/101china.html

2. Ma RCW. Epidemiology of diabetes and diabetic complications in China. Diabetologia 2018;61:1249-60.

3. Serrano N, Villa-Roel C, Gamboa-Delgado EM, et al. Early evaluation of the metabolic syndrome in Bucaramanga, Colombia. Transl Pediatr 2019;8:363-70.

4. Home P, Riddle M, Cefalu WT, et al. Insulin therapy in people with type 2 diabetes: opportunities and challenges? Diabetes Care 2014;37:1499-508.

5. Medical Advisory Secretariat. Continuous Subcutaneous Insulin Infusion (CSII) Pumps for Type 1 and Type 2 Adult Diabetic Populations: An Evidence-Based Analysis. Ont Health Technol Assess Ser 2009;9:1-58.

6. American Diabetes Association. Continuous subcutaneous insulin infusion. Diabetes Care 2004;27 Suppl 1:S110.

7. Karagianni P, Sampanis C, Katsoulis C, et al. Continuous subcutaneous insulin infusion versus multiple daily injections. Hippokratia 2009;13:93-96.

8. Kamoi K, Miyakoshi M, Maruyama R. A quality-oflife assessment of intensive insulin therapy using insulin lispro switched from short-acting insulin and measured by an ITR-QOL questionnaire: a prospective comparison of multiple daily insulin injections and continuous subcutaneous insulin infusion. Diabetes Res Clin Pract 2004;64:19-25.

9. Pickup JC, Renard E. Long-acting insulin analogs versus insulin pump therapy for the treatment of type 1 and type 
2 diabetes. Diabetes Care 2008;31 Suppl 2:S140-5.

10. Pickup J, Mattock M, Kerry S. Glycaemic control with continuous subcutaneous insulin infusion compared with intensive insulin injections in patients with type 1 diabetes: meta-analysis of randomised controlled trials. BMJ 2002;324:705.

11. Bui HD, Jing X, Lu R, et al. Prevalence of and factors related to microvascular complications in patients with type 2 diabetes mellitus in Tianjin, China: a cross-sectional study. Ann Transl Med 2019;7:325.

12. Medtronic Diabetes. Obtain a Good Blood Glucose Control With the Paradigm Real Time System (RTD). Available online: https://clinicaltrials.gov/ct2/show/ NCT00441129

13. Randomized controlled multicnter CSII clinical trial for ATA-I-1-0 insulin pump in the intensive treatment of

Cite this article as: Du J, Zang L, Li Y, Liu J, Wang L, Duan Y, Guo G, Li Y, Zhang Y, Wang G, Mu Y. A multi-center randomized controlled trial of the LenoMed ATA-I-1-0 insulin pump for the intensive treatment of diabetic patients. Ann Transl Med 2020;8(22):1485. doi: 10.21037/atm-20-6024 diabetes patients. Available online: http://www.chictr.org. $\mathrm{cn} /$ showprojen.aspx? proj=49203

14. Xing FX, Chen XT, Zhao HK, et al. Clinical Investigation of Intelligent Insulin Pump and Its Accessories. China Medical Device Information 2011;2011:17-20.

15. Lv XF, Wu JX, Peng Y, et al. Clinical study of insulin pumps made in China in diabetic treatment. Acad J PLA Postgrad Med Sch 2002;23:262-4.

16. Cummins E, Royle P, Snaith A, et al. Clinical effectiveness and cost-effectiveness of continuous subcutaneous insulin infusion for diabetes: systematic review and economic evaluation. Health Technol Assess 2010;14:iii-iv, xi-xvi, $1-181$.

(English Language Editor: C Belazar-Maseh) 